\title{
Spirastrellolide A: Revised Structure, Progress Towards the Relative Configuration, and Inhibition of Protein Phosphatase 2A
}

David E. Williams, ${ }^{\dagger}$ Marshall Lapawa, ${ }^{\S}$ Xidong Feng, ${ }^{\sharp}$ Tamsin Tarling, ${ }^{*}$ Michel Roberg, ${ }^{*}$ and Raymond J. Andersen $^{+^{*}}$

Departments of Chemistry, Earth \& Ocean Sciences, and Biochemistry \& Molecular Biology, University of British Columbia, Vancouver, B.C., CANADA, V6T 1Z1; Wyeth Research, 401 North Middletown Road, Pearl River, New York 10965

\section{Supporting Information}




\section{Page 2}

\section{Index:}

Experimental Section

Table $1-{ }^{1} \mathrm{H}$ NMR Data for $\mathbf{4}$ and $\mathbf{5}$

Table $2-{ }^{13} \mathrm{C}$ NMR Data for $\mathbf{4}$ and 5

Table 3 - NMR data for methylspirastrelloliodepentaacetate

$800 \mathrm{MHz}{ }^{1} \mathrm{H}$ NMR Spectrum for spirastrelloliode A methyl ester (4)

$800 \mathrm{MHz}{ }^{1} \mathrm{H}$ NMR Spectrum of the bisacetonide $\mathbf{5}$ of spirastrellolide methyl ester Page 13

$500 \mathrm{MHz} 1 \mathrm{H}$ NMR Spectrum of the pentaacetate of spirastrellolide A methyl ester Page 14

ESTOFLRMS of Spirastrellolide A methyl ester (4) (MeOH and MeOD)

ESFTHRMS of Spirastrellolide A methyl ester (4)
Pages 3 and 4

Pages 5 and 6

Pages 7 and 8

Pages 9, 10, and 11

Page 12

Page 15

Page 16 


\section{Page 3}

\section{Experimental Section}

\section{Extraction of Spirastrella coccinea and Isolation of Spirastrellolide A Methyl Ester (4):}

Specimens of the red sheet sponge Spirastrella coccinea (Duchassaing \& Michelotti, 1864) were collected by hand using SCUBA on walls at a depth of 2-5 m off Capucin, Guadeloupe Channel, Dominica in June 2002. Freshly collected sponge was frozen on site and transported frozen to Vancouver. A voucher sample has been deposited at the Zoological Museum of Amsterdam (ZMA POR. 16778).

A sample of sponge (19 kg) was cut into small pieces, immersed in and subsequently extracted repeatedly with $\mathrm{MeOH}(3 \times 20 \mathrm{~L})$ at room temperature. The combined $\mathrm{MeOH}$ extracts were concentrated in vacuo and the resultant red gum was then partitioned between EtOAc $(5 \times 1 \mathrm{~L})$ and $\mathrm{H}_{2} \mathrm{O}(6.0 \mathrm{~L})$. The combined EtOAc extracts were evaporated to dryness to give $42 \mathrm{~g}$ of red oil that was partitioned between hexanes (4 x $300 \mathrm{~mL})$ and 4:1 $\mathrm{MeOH} / \mathrm{H}_{2} \mathrm{O}\left(1 \mathrm{~L}\right.$ ). The ratio of the $\mathrm{MeOH}$ extract was adjusted to 2:1 MeOH/ $\mathrm{H}_{2} \mathrm{O}$ (for a total of $1200 \mathrm{~mL}$ ) and extracted with $\mathrm{CH}_{2} \mathrm{Cl}_{2}(4 \times 200 \mathrm{~mL})$. The $\mathrm{CH}_{2} \mathrm{Cl}_{2}$ extracts were combined and evaporated to dryness to give 2.21 $\mathrm{g}$ of a potently active antimitotic amorphous red solid. To facilitate the ease of isolation, the $\mathrm{CH}_{2} \mathrm{Cl}_{2}$ extract was methylated at this stage. Hence, the active material was treated with diazomethane that was generated in-situ by the addition of $10 \mathrm{~mL}$ of $2.0 \mathrm{M}$ trimethylsilyldiazomethane in hexanes to $10 \mathrm{~mL}$ of anhydrous $\mathrm{MeOH}$ in $40 \mathrm{~mL}$ of $\mathrm{C}_{6} \mathrm{H}_{6}$. The reaction mixture was left stirring for 16 hours at room temperature. After evaporation of the reagents, the sample was fractionated with silica gel flash chromatography employing a step gradient from 95:5 hexanes/EtOAc to MeOH. A fraction C (363.7 mg), eluting with EtOAc, and a fraction D (110.6 mg), eluting with $10 \% \mathrm{MeOH} / \mathrm{EtOAc}$, both showed biological activity and these two fractions were each further fractionated on Sephadex LH-20 using 4:1 $\mathrm{MeOH} / \mathrm{CH}_{2} \mathrm{Cl}_{2}$ as the eluent. From both $\mathrm{C}$ and D was obtained a potently active antimitotic fraction (135.1 and $39.2 \mathrm{mg}$, respectively) each consisting of complex mixtures of spirastrellolide methyl esters. HPLC purification followed using a CSC-Inertsil 150A/ODS2, $5 \mu \mathrm{m} 25$ x $0.94 \mathrm{~cm}$ column. Pure spirastrellolide A methyl ester (4) (45.1 mg) was obtained from fraction $\mathrm{C}$ by using $3: 1 \mathrm{MeOH} / \mathrm{H}_{2} \mathrm{O}$ as the reversed-phase HPLC eluent.. 


\section{Spirastrellolide A methyl ester (4):}

Isolated as a clear oil; $[\alpha]^{25} \mathrm{D}+27^{\circ}\left(\mathrm{c} 0.16, \mathrm{CH}_{2} \mathrm{Cl}_{2}\right) ;{ }^{1} \mathrm{H}$ NMR, see Table $1 ;{ }^{13} \mathrm{C}$ NMR, see Table 2; positive ion HRESMS [M+Na] ${ }^{+} m / z 1049.5260$ (calcd for $\mathrm{C}_{53} \mathrm{H}_{83} \mathrm{O}_{17} \mathrm{ClNa}, 1049.5211$ ); HRFTESMS [M+Na] ${ }^{+} \mathrm{m} / z$ 1049.52026(calcd for $\mathrm{C}_{53} \mathrm{H}_{83} \mathrm{O}_{17} \mathrm{ClNa}, 1049.52110$ ).

\section{Preparation of the Pentaacetate of Spirastrellolide A Methyl Ester:}

In $1 \mathrm{~mL}$ of $\mathrm{C}_{5} \mathrm{H}_{5} \mathrm{~N}$ methylspirastrellolide $\mathrm{A}(\mathbf{1})(4.9 \mathrm{mg})$, acetic anhydride $(0.5 \mathrm{~mL})$ and a crystal of DMAP were stirred for $16 \mathrm{~h}$ at $\mathrm{rt}$. After evaporation of the reagents, the sample was fractionated with silica gel flash chromatography employing a step gradient from 95:5 hexanes/EtOAc to EtOAc. A fraction eluting with 1:1 hexanes/EtOAc was further purified by $\mathrm{C}_{18}$ reversed-phase HPLC using a CSC-Inertsil 150A/ODS2, $5 \mu \mathrm{m} 25$ x $0.94 \mathrm{~cm}$ column and $85: 15 \mathrm{MeOH} / \mathrm{H}_{2} \mathrm{O}$ as eluent to yield pure pentaacetate.

\section{Pentaacetate of Spirastrellolide A Methyl Ester:}

Isolated as a clear oil; ${ }^{1} \mathrm{H}$ NMR, see Table $3 ;{ }^{13} \mathrm{C}$ NMR, see Table 3 ; positive ion HRESMS $[\mathrm{M}+\mathrm{Na}]^{+} \mathrm{m} / \mathrm{z}$ 1259.5790 (calcd $\mathrm{C}_{63} \mathrm{H}_{93} \mathrm{O}_{22} \mathrm{ClNa}, 1259.5746$ ).

\section{Preparation of the Bisacetonide 5 of Spirastrellolide A Methyl Ester :}

Methylspirastrellolide A (1) $(7.5 \mathrm{mg})$ was dissolved in $\mathrm{CH}_{2} \mathrm{Cl}_{2}(0.5 \mathrm{~mL})$. To this solution was added dimethoxypropane $(0.5 \mathrm{~mL})$ and a catalytic amount of pyridinium $\rho$-toluene sulfonate. The reaction mixture was stirred for $48 \mathrm{~h}$ at $\mathrm{rt}$. The solvent and excess reagent were evaporated to dryness. The reaction mixture was then purified on HPLC, using a CSC-Inertsil 150A/ODS2, $5 \mu \mathrm{m} 25$ x $0.94 \mathrm{~cm}$ column and 19:1 MeCN/ $\mathrm{H}_{2} \mathrm{O}$ as eluent, to give the bisacetonide $\mathbf{5}$ as a clear oil (3.1 $\mathrm{mg})$.

\section{Bisacetonide 5:}

Isolated as a clear oil; $[\alpha]^{25} \mathrm{D}+5^{\circ}\left(\mathrm{c} 0.96, \mathrm{CH}_{2} \mathrm{Cl}_{2}\right) ;{ }^{1} \mathrm{H} \mathrm{NMR}$, see Table $1 ;{ }^{13} \mathrm{C}$ NMR, see Table 2; positive ion HRESMS [M+Na] ${ }^{+} m / z 1129.5888$ (calcd for $\mathrm{C}_{59} \mathrm{H}_{91} \mathrm{O}_{17} \mathrm{ClNa} 1129.5845$ ). 


\section{Page 5}

Table 1. $800 \mathrm{MHz}{ }^{1} \mathrm{H}$ NMR Data for Spirastrellolide Methyl Ester A (4) and the Bisacetonide 5 recorded in $\mathrm{C}_{6} \mathrm{D}_{6}$.

\begin{tabular}{|c|c|c|c|c|}
\hline Atom \# & ${ }^{1} \mathrm{H}(\delta)$ for 4 & ROESY for $4^{a}$ & ${ }^{1} \mathrm{H}(\delta)$ for bisacetonide 5 & ROESY for bisacetonide $5^{\mathrm{a}}$ \\
\hline $2 \mathrm{a}$ & $2.48 \mathrm{dd} J=17.2,10.0 \mathrm{~Hz}$ & & $2.62 \mathrm{dd} J=16.3,10.0 \mathrm{~Hz}$ & \\
\hline $2 \mathrm{~b}$ & 2.11 & 1.00 & $2.22 \mathrm{dd} J=16.3,1.6 \mathrm{~Hz}$ & \\
\hline $3 \mathrm{ax}$ & $3.68 \mathrm{~m}$ & $1.07,1.24$ & $3.82 \mathrm{~m}$ & $1.27,3.59$ \\
\hline $4_{\mathrm{ax}}$ & 0.95 dddd $J=12.8,12.8,12.8,3.5 \mathrm{~Hz}$ & & 1.06 & 1.13 \\
\hline $4_{\mathrm{eq}}$ & $1.00 \mathrm{~m}$ & 2.11 & 1.06 & \\
\hline $5 \mathrm{ax}$ & 1.24 & $2.53,3.67,3.68$ & 1.27 & $3.59,3.82$ \\
\hline $5_{\mathrm{eq}}$ & 1.50 & & 1.48 & \\
\hline $6 \mathrm{ax}$ & $1.00 \mathrm{~m}$ & & 1.13 & 1.06 \\
\hline $6_{\text {eq }}$ & $1.17 \mathrm{~m}$ & $2.02,4.31$ & $1.30 \mathrm{bd} J=11.2 \mathrm{~Hz}$ & \\
\hline $7 \mathrm{ax}$ & $3.67 \mathrm{~m}$ & $1.07,1.24,2.53$ & $3.59 \mathrm{bt} J=10.0 \mathrm{~Hz}$ & $1.27,1.71,3.82,4.30$ \\
\hline $8 \mathrm{a}$ & 1.52 & 4.70 & $1.46 \mathrm{~m}$ & \\
\hline $8 \mathrm{~b}$ & $2.02 \mathrm{~m}$ & $1.17,4.70$ & 1.66 & \\
\hline 9 & $4.31 \mathrm{~m}$ & 1.17 & $4.30 \mathrm{td} J=10.8,3.2 \mathrm{~Hz}$ & $1.71,3.59$ \\
\hline $10 \mathrm{a}$ & $1.28 \mathrm{ddd} J=14.4,5.8,1.8 \mathrm{~Hz}$ & 1.43 & 1.34 & 1.58 \\
\hline $10_{\mathrm{b}}$ & $1.89 \mathrm{ddd} J=14.4,9.9,3.2 \mathrm{~Hz}$ & 2.09 & 1.69 & \\
\hline 11 & $4.70 \mathrm{bt} J=9.9 \mathrm{~Hz}$ & $1.52,2.02,3.94,4.37$ & $4.55 \mathrm{~m}$ & 1.65 \\
\hline $12 \mathrm{a}$ & $1.43 \mathrm{~m}$ & $1.28,1.94$ & 1.58 ddd $J=12.0,10.4,3.2 \mathrm{~Hz}$ & $1.34,1.92$ \\
\hline $12_{b}$ & $2.09 \mathrm{ddd} J=13.6,9.9,2.4 \mathrm{~Hz}$ & $0.89,1.89$ & $2.17 \mathrm{ddd} J=12.0,10.4,3.2 \mathrm{~Hz}$ & \\
\hline $13_{\mathrm{ax}}$ & $3.94 \mathrm{t} \mathrm{J}=10.0 \mathrm{~Hz}$ & $0.89,4.37,4.70$ & $4.07 \mathrm{td} J=9.6,3.2 \mathrm{~Hz}$ & $1.08,2.26,3.89$ \\
\hline $14 \mathrm{ax}$ & 1.94 & 1.43 & 1.92 & 1.58 \\
\hline 15 & $5.57 \mathrm{dd} J=9.6,1.6 \mathrm{~Hz}$ & 0.89 & $5.56 \mathrm{dd} J=9.9,2.4 \mathrm{~Hz}$ & 1.08 \\
\hline 16 & $5.54 \mathrm{~m}$ & 1.45 & $5.66 \mathrm{dd} J=9.9,2.8 \mathrm{~Hz}$ & $1.49,1.71$ \\
\hline $18 \mathrm{ax}$ & $1.45 \operatorname{td} J=13.6,4.0 \mathrm{~Hz}$ & $3.42,5.54$ & 1.49 & $3.50,5.66$ \\
\hline $18_{\mathrm{eq}}$ & $1.80 \mathrm{dt} J=12.8,3.6 \mathrm{~Hz}$ & & 1.95 & \\
\hline $19 \mathrm{ax}$ & 1.98 & $3.27,4.37$ & 1.94 & $3.16,3.89$ \\
\hline $19 \mathrm{eq}$ & $1.85 \mathrm{~m}$ & 3.27 & $1.85 \mathrm{~m}$ & 3.16 \\
\hline $20_{\mathrm{ax}}$ & 3.42 ddd $J=10.7,10.1,4.8 \mathrm{~Hz}$ & $1.45,3.27,4.16$ & $3.50 \mathrm{td} J=10.0,4.8 \mathrm{~Hz}$ & 1.49, 3.16, 4.66, \\
\hline $21_{\mathrm{ax}}$ & $4.37 \mathrm{~d} J=10.4 \mathrm{~Hz}$ & $1.98,3.27,3.80,3.94,4.70$ & $3.89 \mathrm{~d} J=9.6 \mathrm{~Hz}$ & $1.20,1.94,2.26,3.16,4.07$ \\
\hline 22 & 4.16 & $1.67,2.16,3.27,3.42$ & $4.66 \mathrm{~d} J=6.4 \mathrm{~Hz}$ & $1.20,1.50,3.16,3.50$ \\
\hline 23 & $3.80 \mathrm{bd} J=8.8 \mathrm{~Hz}$ & $1.23,1.67,4.37,4.46$ & $4.00 \mathrm{dd} J=10.4,6.4 \mathrm{~Hz}$ & $1.20,1.50,2.44$ \\
\hline 24 & $2.16 \mathrm{~m}$ & $1.23,4.16$ & $2.26 \mathrm{~m}$ & $1.91,3.89,4.07$ \\
\hline $25 \mathrm{a}$ & 1.37 & 3.94 & 1.18 & 3.75 \\
\hline $25 \mathrm{~b}$ & $2.36 \mathrm{~m}$ & 3.94 & $2.44 \mathrm{~m}$ & \\
\hline $26_{\mathrm{a}}$ & 2.53 & $1.23,3.57$ & $2.57 \mathrm{~m}$ & 3.75 \\
\hline $26_{\mathrm{b}}$ & 1.38 & 1.23 & 1.91 & $1.20,2.26$ \\
\hline $27 \mathrm{ax}$ & $3.94 \mathrm{t} \mathrm{J}=10.0 \mathrm{~Hz}$ & $1.37,2.36,3.83,4.23$ & 4.11 & 3.78 \\
\hline $28 \mathrm{ax}$ & $3.57 \mathrm{t} \mathrm{J}=10.0 \mathrm{~Hz}$ & $2.53,1.36$ & $3.75 \mathrm{t} J=9.6 \mathrm{~Hz}$ & $1.18,1.34,2.44,2.57,3.31$ \\
\hline $29 \mathrm{ax}$ & $3.83 \mathrm{ddd} J=11.2,9.6,5.6 \mathrm{~Hz}$ & $3.35,3.94$ & $3.78 \mathrm{ddd} J=9.6,9 \cdot 6,4.8 \mathrm{~Hz}$ & $3.31,4.11$ \\
\hline
\end{tabular}


Page 6

\begin{tabular}{|c|c|c|c|c|}
\hline $\begin{array}{l}30_{\mathrm{ax}} \\
30_{\mathrm{eq}}\end{array}$ & $\begin{array}{l}1.36 \\
2.11\end{array}$ & $\begin{array}{l}1.70,3.21,3.35,3.57 \\
3.35,1.34\end{array}$ & $\begin{array}{l}1.34 \mathrm{dd} J=12.8,10.4 \mathrm{~Hz} \\
2.14 \mathrm{dd} J=12.8,4.8 \mathrm{~Hz}\end{array}$ & $\begin{array}{l}3.31,3.75 \\
3.31\end{array}$ \\
\hline $32 \mathrm{ax}$ & $1.34 \mathrm{~m}$ & $1.49,2.11$ & $1.37 \mathrm{td} J=13.6,4.0 \mathrm{~Hz}$ & 1.50 \\
\hline $32 \mathrm{eq}$ & $1.70 \mathrm{dt} J=12.8,3.2 \mathrm{~Hz}$ & 1.36 & $1.63 \mathrm{dt} J=13.6,3.2$ & \\
\hline $33_{\mathrm{eq}}$ & 2.13 & 1.07 & 2.01 dddd $J=13.1,13.1,13.1,3.8 \mathrm{H}$ & 1.04 \\
\hline $33 \mathrm{ax}$ & 1.22 & $1.07,5.90$ & $1.18 \mathrm{~m}$ & 1.04 \\
\hline $34 \mathrm{ax}$ & $1.49 \mathrm{~m}$ & 1.34 & 1.50 & $1.37,1.95$ \\
\hline $36_{\mathrm{a}}$ & $2.29 \mathrm{dd} J=15.6,6.8 \mathrm{~Hz}$ & 4.23 & $2.23 \mathrm{dd} J=15.2,6.4 \mathrm{~Hz}$ & 4.18 \\
\hline $36_{\mathrm{b}}$ & $1.98 \mathrm{~d} \mathrm{~J}=15.2 \mathrm{~Hz}$ & 1.07 & $1.95 \mathrm{~d} \mathrm{~J}=15.2 \mathrm{~Hz}$ & $1.04,1.50$ \\
\hline 37 & $5.53 \mathrm{~m}$ & & 5.53 & \\
\hline 38 & $4.23 \mathrm{dt} J=11.2,3.2 \mathrm{~Hz}$ & $2.29,3.94$ & $4.18 \mathrm{dt} J=11.2,2.8 \mathrm{~Hz}$ & 2.23 \\
\hline $39 \mathrm{a}$ & $2.52 \mathrm{~m}$ & 5.90 & $2.78 \mathrm{~m}$ & 5.78 \\
\hline $39 \mathrm{~b}$ & $3.21 \mathrm{~m}$ & $1.36,5.90$ & $2.88 \mathrm{~m}$ & \\
\hline 40 & $5.56 \mathrm{~m}$ & $2.65,2.75$ & 5.52 & 2.82 \\
\hline 41 & $5.90 \mathrm{dt} J=15.2,6.4 \mathrm{~Hz}$ & $1.22,2.52,3.21$ & $5.78 \mathrm{dt} J=16.0,6.4 \mathrm{~Hz}$ & 2.78 \\
\hline $42 \mathrm{a}$ & $2.75 \mathrm{dt} J=15.2,6.9 \mathrm{~Hz}$ & $2.53,5.56$ & $2.82 \mathrm{~m}$ & 5.52 \\
\hline $42 \mathrm{~b}$ & $2.65 \mathrm{dt} J=15.2,7.2 \mathrm{~Hz}$ & 5.56 & $2.82 \mathrm{~m}$ & 5.52 \\
\hline 43 & 5.68 dddt $J=9.9,9.9,9.9,<1.2 \mathrm{~Hz}$ & & $5.69 \mathrm{~m}$ & \\
\hline 44 & 5.45 dddt $J=8.9,8.9,8.9,<1.2 \mathrm{~Hz}$ & & 5.51 & \\
\hline $45_{\mathrm{a}}$ & 2.53 & $1.24,2.75,3.67,4.17$ & $2.51 \mathrm{~m}$ & \\
\hline $45_{b}$ & $2.37 \mathrm{~m}$ & 4.17 & $2.40 \mathrm{~m}$ & \\
\hline 46 & $4.19 \mathrm{~m}$ & & 4.12 & \\
\hline 48 & $0.89 \mathrm{~d} J=7.2 \mathrm{~Hz}$ & $2.09,5.57$ & $1.08 \mathrm{~d} \mathrm{~J}=7.2 \mathrm{~Hz}$ & $4.07,5.56$ \\
\hline 49 & $3.27 \mathrm{~s}$ & $\begin{array}{l}1.23,1.85,1.98,3.42,4.16, \\
4.37\end{array}$ & $3.16 \mathrm{~s}$ & $1.85,1.94,3.50,3.89,4.66$ \\
\hline 50 & $1.23 \mathrm{~d} \mathrm{~J}=7.2 \mathrm{~Hz}$ & $1.38,2.53,3.27,3.80$ & $1.20 \mathrm{~d} \mathrm{~J}=6.4 \mathrm{~Hz}$ & $1.91,3.89,4.00,4.66$ \\
\hline 51 & $3.35 \mathrm{~s}$ & $1.36,2.11,3.83$ & $3.31 \mathrm{~s}$ & $1.34,2.14,3.75,3.78$ \\
\hline 52 & $1.07 \mathrm{~d} J=7.2 \mathrm{~Hz}$ & $1.22,1.98,2.13$ & $1.04 \mathrm{~d} \mathrm{~J}=7.2 \mathrm{~Hz}$ & $1.18,1.95,2.01$ \\
\hline 53 & $3.32 \mathrm{~s}$ & & $3.28 \mathrm{~s}$ & \\
\hline $11 \mathrm{OH}$ & $4.57 \mathrm{bs}$ & & & \\
\hline $22 \mathrm{OH}$ & $1.67 \mathrm{~d} \mathrm{~J}=11.2 \mathrm{~Hz}$ & $3.80,4.16$ & & \\
\hline $23 \mathrm{OH}$ & $4.46 \mathrm{bs}$ & 3.80 & & \\
\hline $46 \mathrm{OH}$ & 4.17 & & $3.00 \mathrm{~d} J=6.4 \mathrm{~Hz}$ & \\
\hline 55 & & & $1.65 \mathrm{~s}$ & $1.71,4.55$ \\
\hline 56 & & & $1.71 \mathrm{bs}$ & $1.65,3.59,4.30$ \\
\hline 58 & & & $1.50 \mathrm{~s}$ & 1.71, 4.00, 4.66, \\
\hline 59 & & & $1.71 \mathrm{bs}$ & 1.50 \\
\hline
\end{tabular}

${ }^{a}$ Chemical shift of proton resonances correlated to the proton resonance listed in the $\delta{ }^{1} \mathrm{H}$ column. Correlations that might result from geminal or vicinal COSY coupling are not included. The ROESY mixing time was $200 \mathrm{~ms}$. Only positive 'ROESY' correlations are quoted. 


\section{Page 7}

Table 2. ${ }^{13} \mathrm{C}$ NMR Data for Spirastrellolide A Methyl Ester (4) and the Bisacetonide 5 recorded in $\mathrm{C}_{6} \mathrm{D}_{6}$.

\begin{tabular}{|c|c|c|}
\hline Atom \# & $4^{a}$ & $5^{b}$ \\
\hline 1 & 169.2 & 169.4 \\
\hline 2 & 42.7 & 43.9 \\
\hline 3 & 74.3 & 73.3 \\
\hline 4 & 31.2 & 32.1 \\
\hline 5 & 24.0 & 24.2 \\
\hline 6 & 30.2 & 32.2 \\
\hline 7 & 76.3 & 76.2 \\
\hline 8 & 42.1 & 42.2 \\
\hline 9 & 66.3 & 64.5 \\
\hline 10 & 44.9 & 39.7 \\
\hline 11 & 64.9 & 62.4 \\
\hline 12 & 42.4 & 44.4 \\
\hline 13 & 71.3 & 69.6 \\
\hline 14 & 35.2 & 36.3 \\
\hline 15 & 135.2 & 132.9 \\
\hline 16 & 129.0 & 129.6 \\
\hline 17 & 93.8 & 92.8 \\
\hline 18 & 34.5 & $34.3^{1}$ \\
\hline 19 & 24.6 & 23.2 \\
\hline 20 & 74.3 & 75.1 \\
\hline 21 & 71.4 & 72.1 \\
\hline 22 & 69.2 & 76.4 \\
\hline 23 & 75.1 & 82.3 \\
\hline 24 & 34.0 & $34.2^{1}$ \\
\hline 25 & 26.3 & 24.8 \\
\hline 26 & 31.5 & 28.0 \\
\hline 27 & 75.1 & $72.6^{2}$ \\
\hline 28 & 65.9 & 62.4 \\
\hline 29 & 79.3 & 79.9 \\
\hline 30 & 44.1 & 43.5 \\
\hline 31 & 97.6 & 98.1 \\
\hline 32 & 36.5 & 36.5 \\
\hline 33 & 24.1 & 23.9 \\
\hline 34 & 38.7 & 38.3 \\
\hline 35 & 108.7 & 108.5 \\
\hline 36 & 47.0 & 46.9 \\
\hline 37 & 73.3 & 72.9 \\
\hline
\end{tabular}


Page 8

\begin{tabular}{|c|c|c|}
\hline 38 & 84.0 & 83.4 \\
\hline 39 & 30.4 & 32.4 \\
\hline 40 & 126.1 & 126.0 \\
\hline 41 & 131.8 & 133.1 \\
\hline 42 & 31.0 & 31.2 \\
\hline 43 & 131.8 & 131.3 \\
\hline 44 & 124.4 & 124.4 \\
\hline 45 & 32.8 & 32.6 \\
\hline 46 & 70.6 & $70.4^{2}$ \\
\hline 47 & 174.8 & 175.0 \\
\hline 48 & 16.8 & 18.3 \\
\hline 49 & 56.7 & 56.2 \\
\hline 50 & 18.5 & 18.8 \\
\hline 51 & 57.5 & 57.6 \\
\hline 52 & 17.0 & 16.5 \\
\hline 53 & 51.5 & 51.8 \\
\hline 54 & & 100.8 \\
\hline 55 & & 25.6 \\
\hline 56 & & 27.9 \\
\hline 57 & & 108.7 \\
\hline 58 & & 26.6 \\
\hline 59 & & 27.1 \\
\hline
\end{tabular}

${ }^{\mathrm{a}}$ Recorded at $200 \mathrm{MHz}$.

${ }^{\mathrm{b}}$ Recorded at $100 \mathrm{MHz}$.

1,2 Assignments within a column are interchangeable. 


\section{Page 9}

Table 3. ${ }^{1} \mathrm{H}$ and ${ }^{13} \mathrm{C}$ NMR Data for the Pentaacetate of Spirastrellolide A Methyl Ester recorded in $\mathrm{C}_{6} \mathrm{D}_{6}$.

\begin{tabular}{|c|c|c|}
\hline Atom \# & 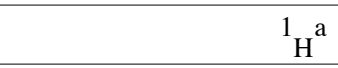 & ${ }^{13} \mathrm{C}$ \\
\hline 1 & & 169.1 \\
\hline 2 & $\begin{array}{l}2.12 \\
2.41 \mathrm{dd} J=15.7,10.3 \mathrm{~Hz}\end{array}$ & 43.0 \\
\hline 3 & $3.65 \mathrm{~m}$ & 74.3 \\
\hline 4 & $\begin{array}{l}1.05 \mathrm{~m} \\
1.05 \mathrm{~m}\end{array}$ & $31.1^{1}$ \\
\hline 5 & $\begin{array}{l}1.25 \\
1.58 \mathrm{~m}\end{array}$ & 23.8 \\
\hline 6 & $\begin{array}{l}1.42 \\
1.58 \mathrm{~m}\end{array}$ & 30.3 \\
\hline 7 & $3.42 \mathrm{~m}$ & 76.3 \\
\hline 8 & $\begin{array}{l}1.97 \\
2.00\end{array}$ & 42.3 \\
\hline 9 & $5.48^{2}$ & $66.8^{2}$ \\
\hline 10 & $\begin{array}{l}1.31 \\
2.07\end{array}$ & 43.3 \\
\hline 11 & $5.46^{2}$ & $67.0^{2}$ \\
\hline 12 & $\begin{array}{l}1.98 \\
2.21\end{array}$ & 40.2 \\
\hline 13 & $3.88 \mathrm{~m}$ & 71.1 \\
\hline 14 & $2.18 \mathrm{~m}$ & 33.8 \\
\hline 15 & 5.46 & 133.8 \\
\hline 16 & 5.56 & 128.6 \\
\hline 17 & & 93.7 \\
\hline 18 & $\begin{array}{l}1.44 \\
1.71\end{array}$ & 34.2 \\
\hline 19 & $\begin{array}{l}1.82 \\
1.82\end{array}$ & 23.6 \\
\hline 20 & $2.98 \mathrm{ddd} J=9.9,9.9,5.1 \mathrm{~Hz}$ & 74.3 \\
\hline 21 & $4.13 \mathrm{~d} J=9.9 \mathrm{~Hz}$ & 71.4 \\
\hline 22 & $5.90 \mathrm{~d} J=5.5 \mathrm{~Hz}$ & 71.4 \\
\hline 23 & 5.55 & 75.0 \\
\hline 24 & 1.75 & 35.5 \\
\hline 25 & $\begin{array}{l}1.30 \\
2.27\end{array}$ & 27.7 \\
\hline 26 & $\begin{array}{l}1.29 \\
2.34\end{array}$ & 31.2 \\
\hline 27 & 3.82 & 73.8 \\
\hline
\end{tabular}


Page 10

\begin{tabular}{|c|c|c|}
\hline 28 & $3.51 \mathrm{t} \mathrm{J}=9.8 \mathrm{~Hz}$ & 65.4 \\
\hline 29 & 3.82 & 79.0 \\
\hline 30 & $\begin{array}{l}1.31 \\
2.10 \\
\end{array}$ & 39.7 \\
\hline 31 & & 97.8 \\
\hline 32 & $\begin{array}{l}1.33 \\
1.73 \\
\end{array}$ & 36.5 \\
\hline 33 & $\begin{array}{l}1.25 \\
2.16 \\
\end{array}$ & 24.2 \\
\hline 34 & $1.48 \mathrm{~m}$ & 38.5 \\
\hline 35 & & 108.4 \\
\hline 36 & $\begin{array}{l}1.90 \\
2.23 \mathrm{~m}\end{array}$ & 47.4 \\
\hline 37 & 5.49 & 72.3 \\
\hline 38 & 4.13 & 83.8 \\
\hline 39 & $\begin{array}{l}2.59 \mathrm{~m} \\
2.65 \mathrm{~m}\end{array}$ & $31.0^{1}$ \\
\hline 40 & $5.63 \mathrm{~m}$ & 127.2 \\
\hline 41 & $5.72 \mathrm{~m}$ & 132.1 \\
\hline 42 & $\begin{array}{l}2.70 \mathrm{~m} \\
2.70 \mathrm{~m}\end{array}$ & 30.8 \\
\hline 43 & 5.58 & 131.0 \\
\hline 44 & 5.46 & 124.3 \\
\hline 45 & $\begin{array}{l}2.54 \mathrm{~m} \\
2.54 \mathrm{~m}\end{array}$ & 29.3 \\
\hline 46 & $5.13 \mathrm{dd} J=7.3,5.3 \mathrm{~Hz}$ & 72.2 \\
\hline 47 & & 170.0 \\
\hline 48 & $0.87 \mathrm{~d} J=7.2 \mathrm{~Hz}$ & 17.2 \\
\hline 49 & $3.14 \mathrm{~s}$ & 56.2 \\
\hline 50 & $1.15 \mathrm{~d} \mathrm{~J}=6.7 \mathrm{~Hz}$ & 17.0 \\
\hline 51 & $3.32 \mathrm{~s}$ & 57.4 \\
\hline 52 & $0.98 \mathrm{~d} J=6.7 \mathrm{~Hz}$ & 16.6 \\
\hline 53 & $3.37 \mathrm{~s}$ & 51.8 \\
\hline $9 \underline{\mathrm{COCH}}_{3}$ & & $170.3^{3}$ \\
\hline $9 \mathrm{COCH}_{3}$ & $2.04 \mathrm{~s}^{2}$ & $21.4^{4}$ \\
\hline $11 \underline{\mathrm{COCH}}_{3}$ & & $169.9^{3}$ \\
\hline $11 \mathrm{COCH}$ & $1.77 \mathrm{~s}^{2}$ & $20.3^{4}$ \\
\hline $22 \mathrm{COCH}_{3}$ & & 169.7 \\
\hline $22 \mathrm{COCH}_{3}$ & $1.81 \mathrm{~s}$ & 20.6 \\
\hline $23 \underline{\mathrm{COCH}}_{3}$ & & 170.0 \\
\hline $23 \mathrm{COCH}_{3}$ & $2.14 \mathrm{~s}$ & 21.5 \\
\hline
\end{tabular}




\section{Page 11}

\begin{tabular}{|c|c|c|}
\hline $46 \mathrm{COCH}_{3}$ & & 170.0 \\
\hline $46 \mathrm{COCH} \underline{-1}_{3}$ & $1.91 \mathrm{~s}$ & 21.1 \\
\hline
\end{tabular}

${ }^{\mathrm{a}}$ Recorded at $500 \mathrm{MHz}$.

${ }^{\mathrm{b}}$ Recorded at $100 \mathrm{MHz}$.

${ }_{1,2,3,4}$ Assignments within a column are interchangeable 
Page 12

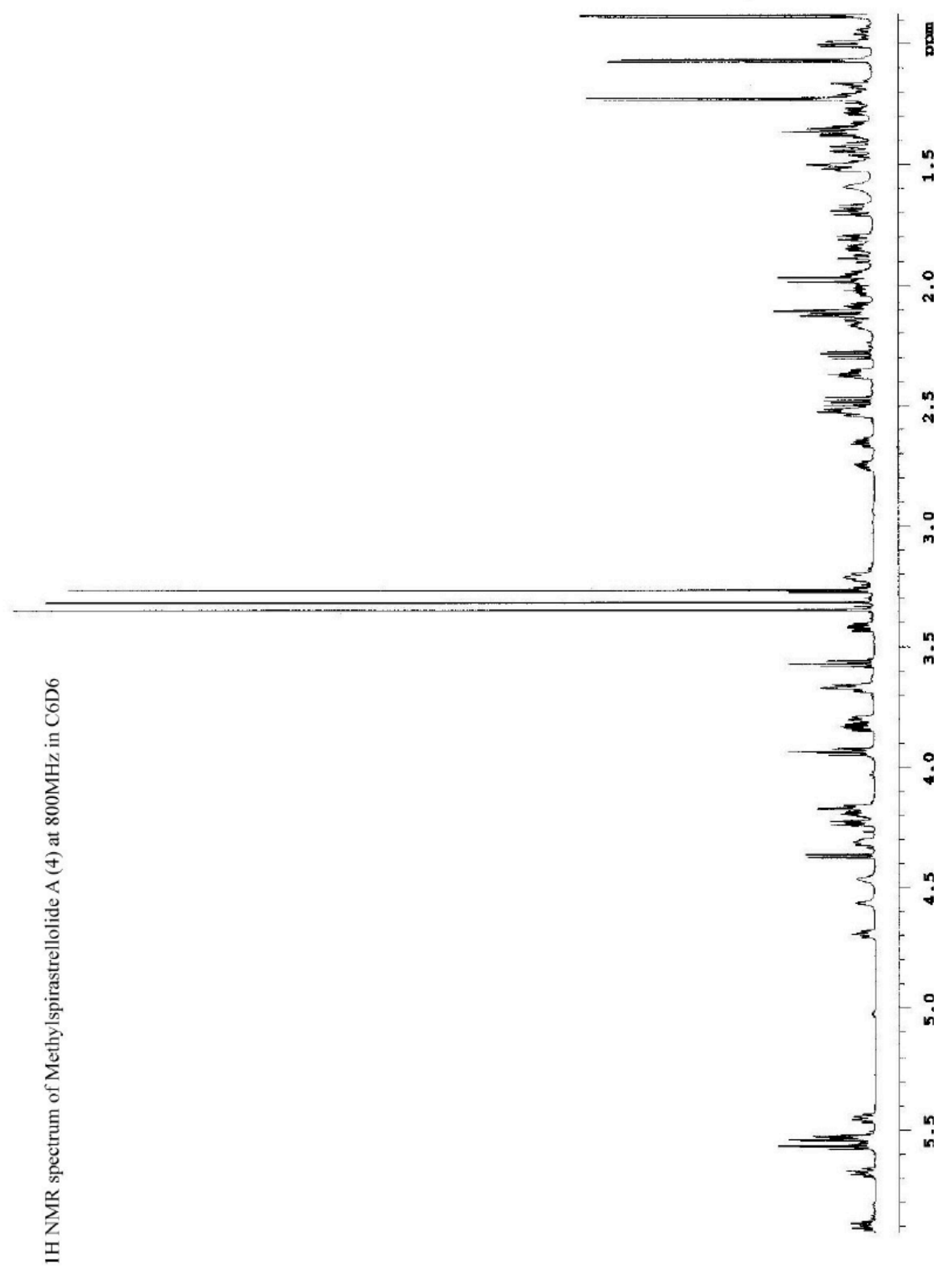


Page 13

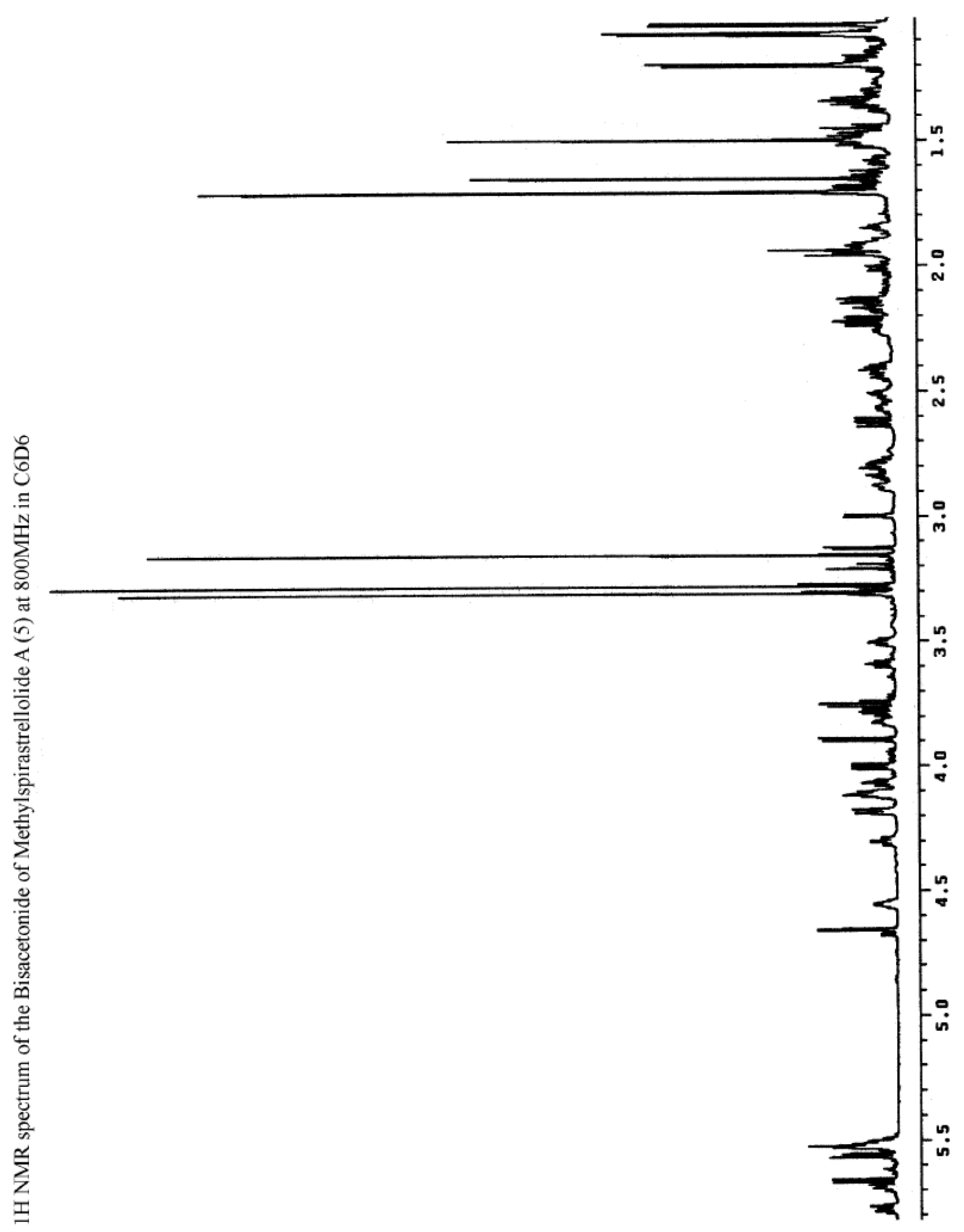


Page 14

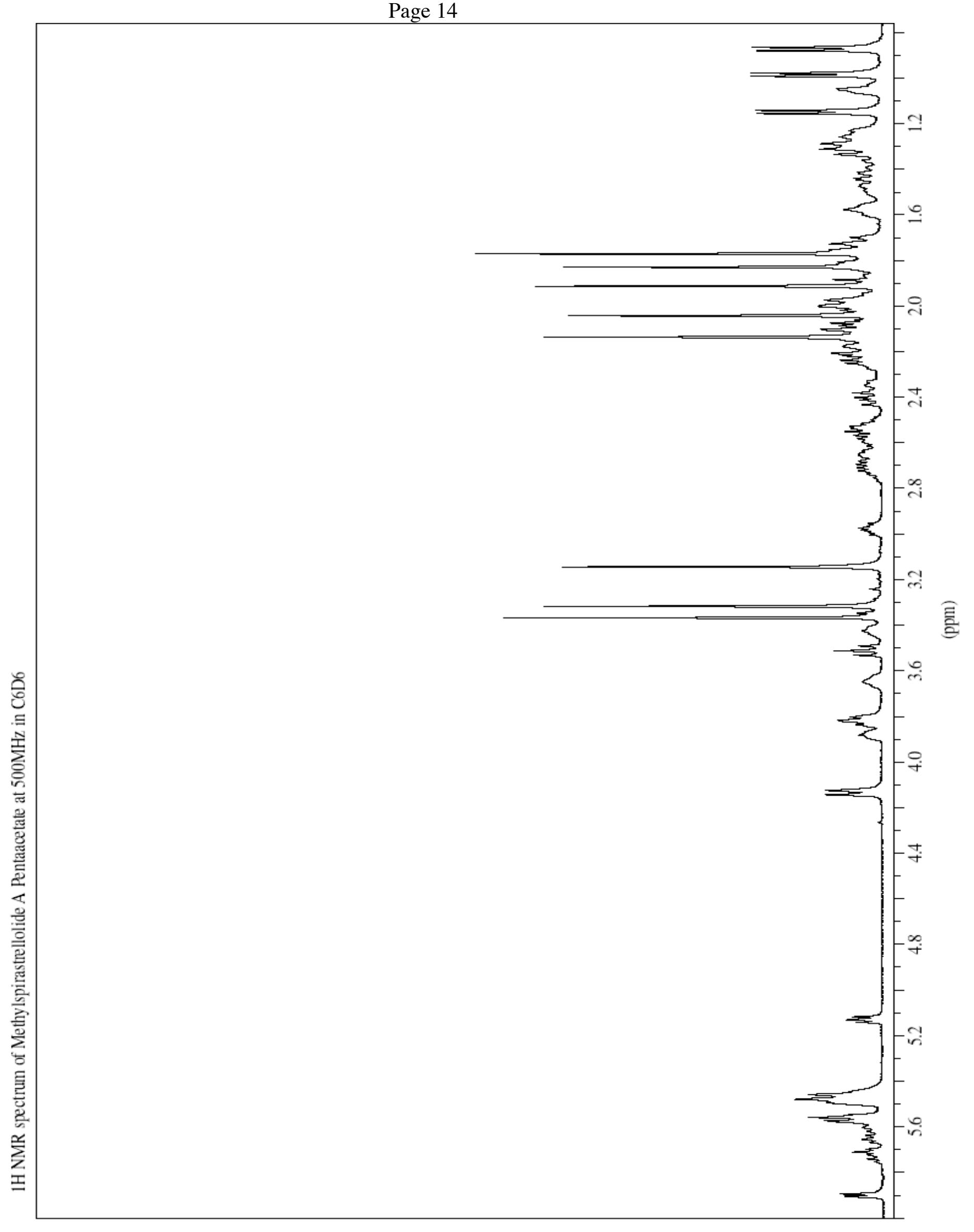




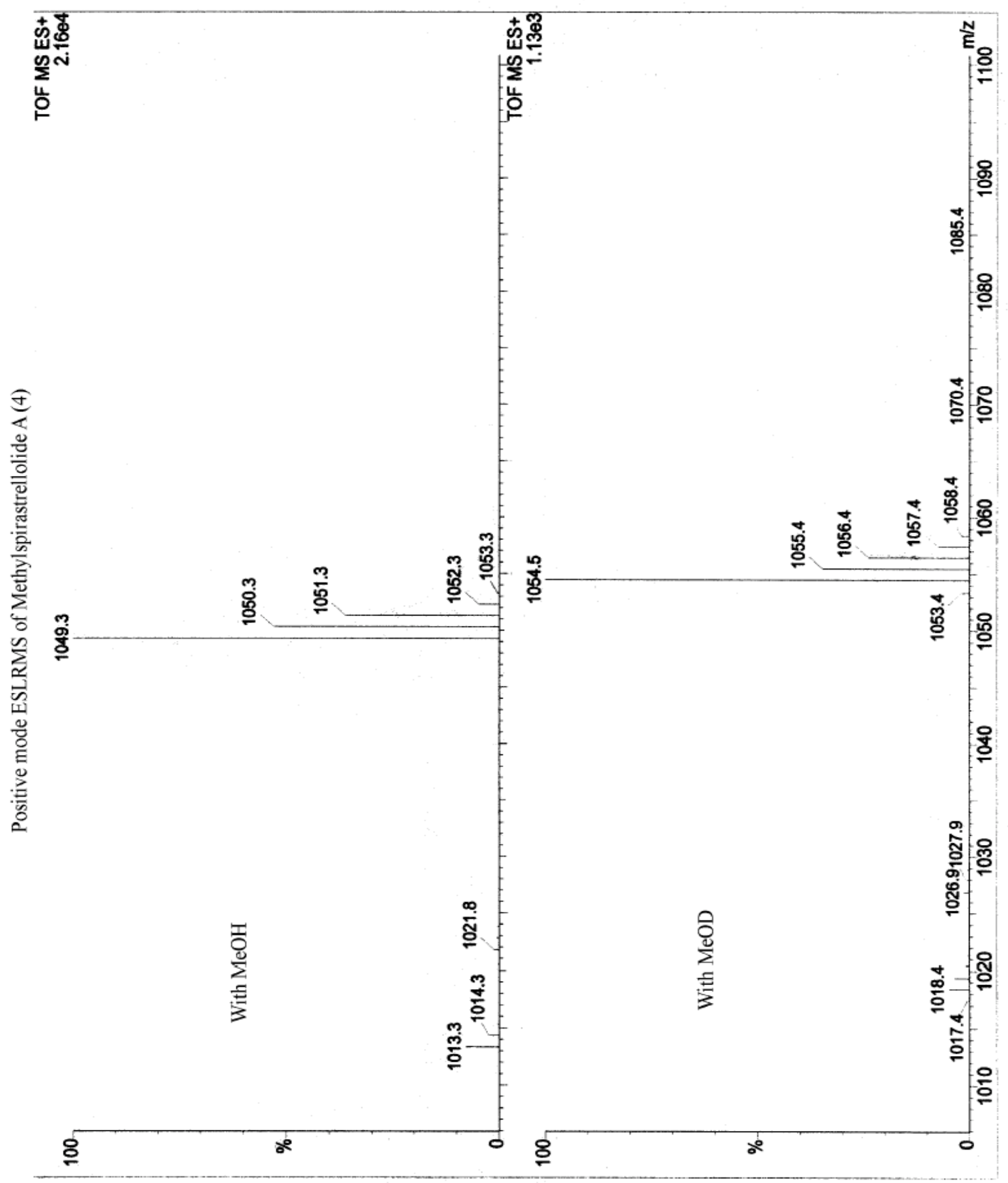


Page 16

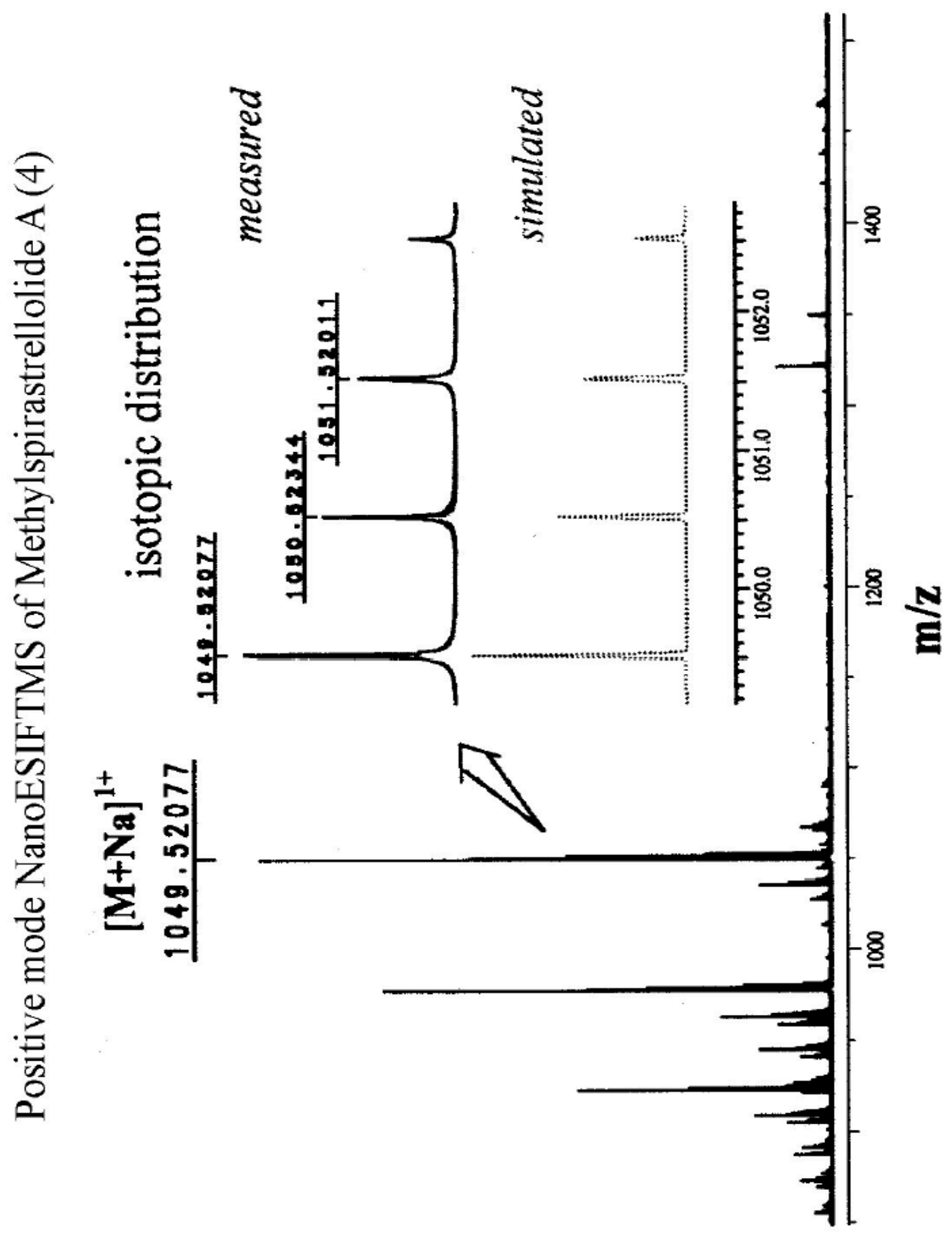

